\title{
Loss of capsule among Streptococcus suis isolates from porcine endocarditis and its biological significance
}

\author{
Nattakan Lakkitjaroen, ${ }^{1}$ Daisuke Takamatsu, ${ }^{2,3}$ Masatoshi Okura, ${ }^{2}$ \\ Masumi Sato, ${ }^{4}$ Makoto Osaki ${ }^{2} \dagger$ and Tsutomu Sekizaki ${ }^{1}$ \\ ${ }^{1}$ Research Center for Food Safety, Graduate School of Agricultural and Life Sciences, \\ University of Tokyo, Tokyo 113-8657, Japan \\ ${ }^{2}$ Research Division of Bacterial and Parasitic Diseases, National Institute of Animal Health, \\ National Agriculture and Food Research Organization, Tsukuba, Ibaraki 305-0856, Japan \\ ${ }^{3}$ United Graduate School of Veterinary Sciences, Gifu University, Gifu 501-1193, Japan \\ ${ }^{4}$ Epidemiological Information Section, National Institute of Animal Health, National Agriculture and \\ Food Research Organization, Tsukuba, Ibaraki 305-0856, Japan
}

Correspondence

Tsutomu Sekizaki

asekizak@mail.ecc.u-tokyo.ac.jp

Daisuke Takamatsu

p1013dt@affrc.go.jp

Received 26 May 2011

Accepted 15 July 2011

\begin{abstract}
Streptococcus suis, particularly serotype 2, is a pathogen of both pigs and humans associated with a wide range of diseases, including meningitis, septicaemia and endocarditis. Among the genes in the capsular polysaccharide biosynthesis (cps) locus, cps2 J exists only in the serotype 2 and $1 / 2$ strains; therefore, cps2J-positive strains are suspected to have capsules of serotype 2 or 1/2. Coagglutination using antiserotype 1 and antiserotype 2 sera and/or transmission electron microscopy analysis of 288 cps $2 \mathrm{~J}$-positive isolates from pigs showed that $32(100 \%)$ isolates from meningitis were encapsulated, whereas 86 (34\%) of 256 isolates from endocarditis were unencapsulated, indicating that capsule loss often occurred in the isolates from endocarditis. To investigate the genetic backgrounds, we randomly selected 43 unencapsulated isolates and analysed their cps loci by PCR scanning. Among them, 8 and 10 isolates apparently had deletions and insertions, respectively, in cps loci. In addition, a representative unencapsulated isolate and an unencapsulated strain showed adherence to porcine and human platelets, a major virulence determinant for infective endocarditis, to a significantly greater extent than the encapsulated strains. Although the capsule is considered to be an important virulence factor in

$S$. suis, these results suggest that loss of capsular production is beneficial to $S$. suis in the course of infective endocarditis.
\end{abstract}

\section{INTRODUCTION}

Streptococcus suis is a zoonotic pathogen that causes serious diseases, including meningitis, arthritis, septicaemia and

†Present address: Agriculture, Forestry and Fisheries Research Council Secretariat, Ministry of Agriculture, Forestry and Fisheries, Chiyoda-ku, Tokyo 100-8950, Japan.

Abbreviations: CcpA, catabolite control protein A; GBS, group B Streptococcus; IS, insertion sequence; TEM, transmission electron microscopy.

The GenBank/EMBL/DDBJ accession numbers for the nucleotide sequence data reported in this paper are AB627103, AB627104, AB627105, AB627106, AB627107, AB627108, AB627109, $A B 627110, A B 627111$ and $A B 627112$ for the inserted sequences of S. suis NL85, NL155, NL171, NL176, NL179, NL184, NL194, NL198, NL201 and NL255, respectively.

Two supplementary tables are available with the online version of this paper. endocarditis, in swine and humans (Gottschalk et al., 2007). S. suis strains are classified into more than 30 serotypes according to the different antigenicity of their capsular polysaccharides (Higgins \& Gottschalk, 2006). Among them, serotype 2 has been predominantly isolated from both infected pigs and human patients in many countries (Higgins \& Gottschalk, 2006; Wertheim et al., 2009). Production of S. suis capsule is mediated by capsular polysaccharide biosynthesis ( $c p s)$ genes clustered in a single locus of the genome. Among the genes in the cps loci identified to date (Holden et al., 2009; Smith et al., 1999a, b, c, 2000), cps2J has been found only in strains of serotypes 2 and $1 / 2$ (the serotype reacting with both antiserotypes 1 and 2 sera); therefore, this gene is sometimes used as a molecular marker of the two serotypes (Smith et al., 1999c). However, for determination of the serotypes, it is necessary to verify their phenotypes by serotype-specific antisera. 
Endocarditis caused by S. suis is often found in adult pigs, particularly in slaughterhouses. In Japan, most of the endocarditis isolates are cps2J positive by PCR (our unpublished observation), suggesting that they are serotype 2 or $1 / 2$. However, more than $50 \%$ of the $S$. suis isolates from porcine endocarditis were shown to be untypable by agglutination tests (Katsumi et al., 1997), although a high percentage of isolates from meningitis and pneumonia were serotypable (Kataoka et al., 1993). Although the polysaccharide capsule is believed to be essential for the virulence of S. suis (Benga et al., 2008; Chabot-Roy et al., 2006; Charland et al., 1998; Smith et al., 1999a), these observations imply that many endocarditis isolates, especially those of serotypes 2 and $1 / 2$, frequently lose their ability to synthesize capsules.

To confirm this speculation, we investigated the capsule production of cps2J-positive isolates from porcine endocarditis and meningitis. Furthermore, the genetic backgrounds of several unencapsulated isolates and the biological significance of unencapsulation were examined. Here we show that capsule loss often occurred in cps $2 J-$ positive endocarditis isolates and that unencapsulation increased the ability of the bacteria to adhere to platelets, which is thought to be a major virulence determinant in the pathogenesis of infective endocarditis.

\section{METHODS}

Bacterial strains and growth conditions. A total of 288 cps2Jpositive $S$. suis isolates from different pigs were used in this study. Among them, 256 isolates were from heart valve vegetations of pigs with endocarditis in regional diagnostic centres in Japan between 1994 and 2009, and 32 isolates, including the well-characterized strain P1/7 (Slater et al., 2003), were from pigs with meningitis. Except P1/7, all of the meningitis isolates were isolated in regional diagnostic centres or the National Institute of Animal Health in Japan between 1989 and 2006. All isolates were stored in Luria-Bertani (Becton Dickinson) broth containing $30 \%$ glycerol at $-80{ }^{\circ} \mathrm{C}$ and minimally passaged for the experiments to avoid changing key traits including capsule production. In addition, S. suis strains S735 (NCTC 10234; serotype 2 reference strain) and 204 (serotype 1 field isolate) (Sekizaki et al., 2001) were used for the production of rabbit antiserum, and $S$. suis strain 89/1591, isolated from a pig with septicaemia (Salasia et al., 1995), and its isogenic unencapsulated mutant (CPS2B) (Okura et al., 2011) were used to compare their phenotypic characteristics with other encapsulated and unencapsulated cps2J-positive isolates. Enterococcus faecalis NCTC 775 was used as a control for PCR scanning analysis. Identification of $S$. suis field isolates was confirmed by species-specific PCR for S. suis (Okwumabua et al., 2003) and/or sequencing of the $16 \mathrm{~S}$ rRNA gene. The presence of cps $2 J$ was examined by PCR as described previously (Silva et al., 2006). Bacteria were cultured in Todd-Hewitt broth (THB; Difco Laboratories, Becton Dickinson) or agar (THA) at $37{ }^{\circ} \mathrm{C}$ in air plus $5 \% \mathrm{CO}_{2}$ for $16 \mathrm{~h}$, unless otherwise indicated. For strain CPS2B, of which the $\operatorname{cps} 2 B$ gene was disrupted by the insertion of a suicide vector containing a spectinomycin resistance gene, spectinomycin $\left(100 \mu \mathrm{g} \mathrm{ml}^{-1}\right)$ was added to the medium.

Production of rabbit antisera. Rabbit antiserotype 1 and antiserotype 2 polyclonal sera were prepared by immunizing rabbits with formalin-killed S. suis strains 204 and S735, respectively, according to the procedure of Higgins \& Gottschalk (1990). Briefly, rabbits weighing $3 \mathrm{~kg}$ were given three injections per week of increasing numbers of bacteria for 4 weeks as follows: first week, 2$4 \times 10^{9}$ c.f.u.; second to fourth week, $4-8 \times 10^{9}$ c.f.u.. Ten days after the last injection, blood samples were collected and the sera were evaluated by coagglutination tests as described below. All animal procedures were carried out according to the regulations and guidelines approved by the Animal Ethics Committee of the National Institute of Animal Health.

Serotyping. The capsular antigens of all cps2J-positive isolates cultured on THA plates were extracted by autoclaving the cells in Dulbecco's PBS (DPBS) at $121{ }^{\circ} \mathrm{C}$ for $15 \mathrm{~min}$ and tested with antiserotype 1 and 2 sera. The coagglutination technique was applied as previously described (Gottschalk et al., 1989; Han et al., 2001). The reaction was judged as positive when agglutination occurred within $5 \mathrm{~min}$. For isolates with a negative agglutination reaction, further coagglutination tests were performed to verify the absence of capsule using isolates subcultured under the conditions recommended to enhance capsular production (Gottschalk et al., 1993).

Transmission electron microscopy (TEM). The samples were prepared according to previous studies (Jacques et al., 1990; Mackie et al., 1979) with some modifications. Briefly, bacterial cells were harvested from cultures on THA plates, washed with PBS $(0.01 \mathrm{M}$, $\mathrm{pH}$ 7.2), and incubated with antiserotype 2 serum at $4{ }^{\circ} \mathrm{C}$ for $1 \mathrm{~h}$. The cells were then washed with deionized distilled water (DDW) and fixed with $5 \%(\mathrm{v} / \mathrm{v})$ glutaraldehyde containing $0.15 \% \quad(\mathrm{w} / \mathrm{v})$ ruthenium red at room temperature for $2 \mathrm{~h}$. The cells were immobilized in $1 \%$ agar, post-fixed with $1 \%$ osmium tetroxide at $4{ }^{\circ} \mathrm{C}$ for $1.5 \mathrm{~h}$, and washed once with DDW. Samples were then dehydrated with a graded series of ethanol and embedded in lowviscosity resin (Quetol 651 mixture; Nisshin EM). Ultrathin sections were stained with uranyl acetate and lead citrate prior to examination with a transmission electron microscope (H-7500; Hitachi).

PCR scanning of genes in the cps loci and sequencing of mutated regions. Twenty-one primer sets covering the whole $c p s$ locus of serotype 2 (Table 1, Fig. 1) were designed on the basis of the $S$. suis strain P1/7 genome sequence data (accession number AM946016). Chromosomal DNA extracted by standard procedures (Mogollon et al., 1990) was used as template DNA, and PCRs were performed using Ex Taq polymerase (Takara Bio) according to the manufacturer's instructions. The conditions of the PCR scanning assay consisted of pre-denaturing at $95{ }^{\circ} \mathrm{C}$ for $5 \mathrm{~min}, 30$ cycles of $20 \mathrm{~s}$ at $95{ }^{\circ} \mathrm{C}, 10 \mathrm{~s}$ at $55{ }^{\circ} \mathrm{C}, 1 \mathrm{~min}$ at $72{ }^{\circ} \mathrm{C}$, and final extension at $72{ }^{\circ} \mathrm{C}$ for 2 min. Chromosomal DNA of S. suis P1/7 and E. faecalis NCTC 775 was used as positive and negative controls, respectively. PCR products were analysed by electrophoresis on $1 \%$ agarose gel and/or a MultiNA microchip electrophoresis system (Shimadzu Biotech).

The PCR products of sizes different from those of S. suis P1/7 were purified by a QIAquick PCR purification kit (Qiagen) following the manufacturer's instructions and sequenced by primer walking. Additional PCR and inverse PCR (Ochman et al., 1988) using different combinations of primers were performed to amplify the altered $c p s$ regions. The PCR products were sequenced by a BigDye terminator v3.1 cycle sequencing kit using a 3130xl Genetic Analyzer (Applied Biosystems). Sequencher Ver. 4.8 (Hitachi Software Engineering) and Artemis software (Wellcome Trust Sanger Institute, http://www.sanger.ac.uk) were used for assembly and analysis of the sequences. Deletions and insertions were determined using the CLUSTAL W Ver. 1.83 (http://clustalw.ddbj.nig.ac.jp/top-e.html) and BLAST (http://blast.ncbi.nlm.nih.gov/Blast.cgi) programs. In addition, IS Finder (http://www-is.biotoul.fr/) was used to annotate and classify insertion sequence (IS) elements. 
Table 1. Primer sets for PCR scanning

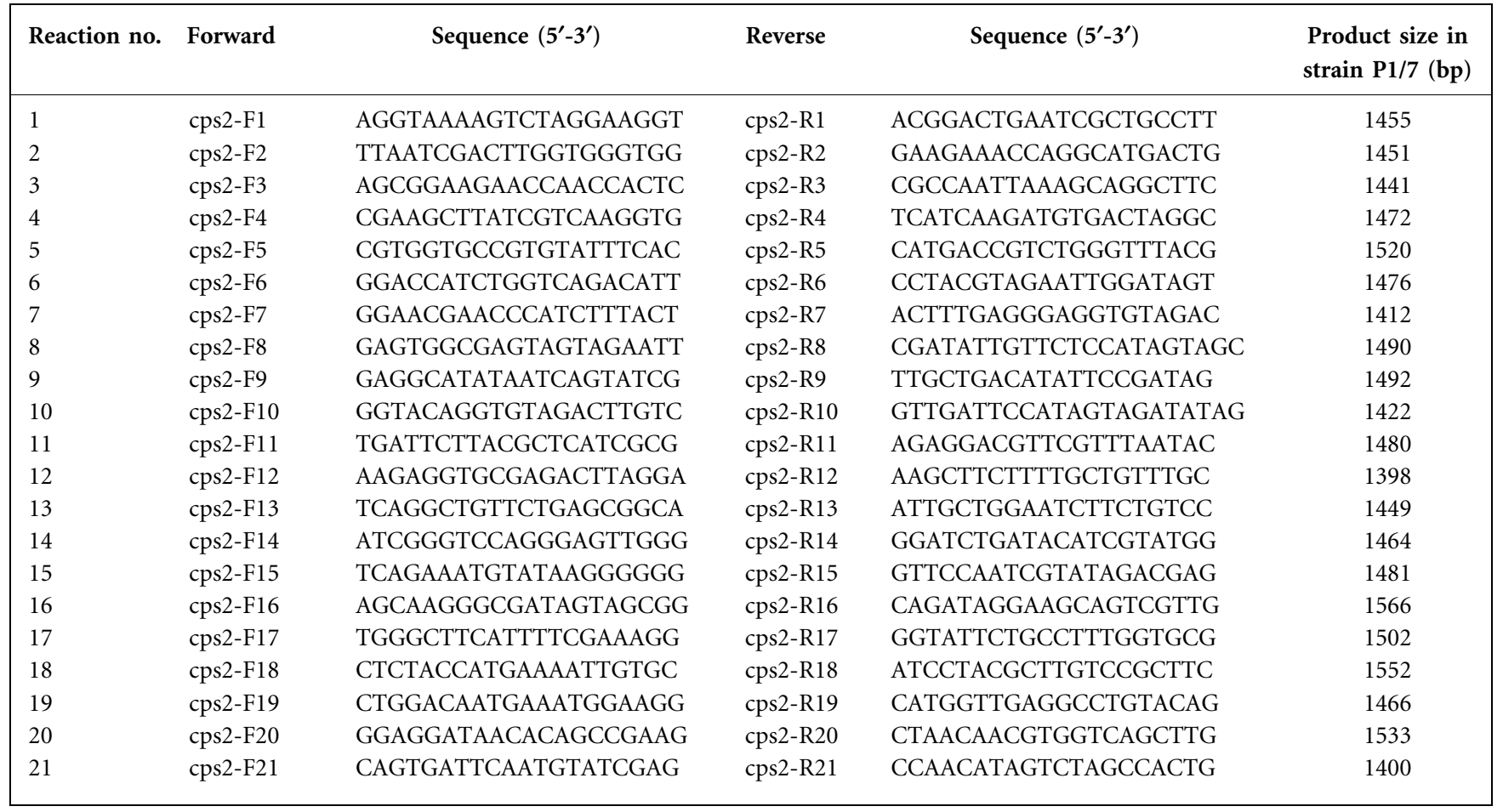

Bacterial adherence to porcine and human platelets. The ability of encapsulated and unencapsulated strains to adhere to porcine and human platelets was evaluated according to a previous study (Hoshinoo et al., 2009) with appropriate modifications. For preparation of the inocula, S. suis strains were cultured in THB or THB with $100 \mu \mathrm{g}$ spectinomycin $\mathrm{ml}^{-1}$ (for strain CPS2B) until the $\mathrm{OD}_{600}$ reached 0.8 . The cultured bacteria were washed twice with DPBS, sonicated in DPBS for $30 \mathrm{~s}$ to disperse the bacterial cells, and then diluted with DPBS to approximately $2 \times 10^{9}$ c.f.u. $\mathrm{ml}^{-1}$. The bacterial suspensions were additionally diluted in triplicate and each dilution was plated twice onto THA to examine the exact concentration of the inocula each time.

Porcine venous blood was freshly obtained from healthy adult pigs kept in the National Institute of Animal Health, and the platelets were prepared by centrifuging the blood at $100 \mathrm{~g}$ for $15 \mathrm{~min}$ and collecting the upper layer. Human platelets donated for transfusion were obtained from the Japan Red Cross Society. The collected platelets were washed twice with platelet wash buffer $[0.14 \mathrm{M} \mathrm{NaCl}, 20 \mathrm{mM}$ HEPES, 1 mM EDTA ( $\mathrm{pH}$ 6.6) containing $1 \mu \mathrm{g}$ prostaglandin $\mathrm{I}_{2} \mathrm{ml}^{-1}$ for the first wash], fixed with $0.8 \%$ formalin in DPBS, and immobilized in eight-well culture slides (BD Falcon glass; Becton Dickinson) coated with $0.01 \%$ poly-L-lysine solution at approximately $1 \times 10^{8}$ platelets per well. The wells were treated with
$1 \times$ blocking reagent (Roche Applied Science) for $1 \mathrm{~h}$ with gentle rocking at room temperature to minimize non-specific adherence. After removal of the blocking reagent by aspiration, $500 \mu \mathrm{l}$ of each bacterial suspension was inoculated into the wells to give an m.o.i. of approximately 10. After incubation with gentle shaking for $2 \mathrm{~h}$ at room temperature, platelets were washed four times with DPBS to remove the unattached bacteria, fixed with pre-chilled methanol, and stained with $5 \%$ Giemsa solution. The numbers of bacterial cells attached to 100 platelets were determined by light microscopy. In each strain/isolate, the assay was repeated six times using porcine platelets from two different pigs and twelve times using human platelets of three different lots. The differences were analysed by Student's unpaired $t$-test at $95 \%$ confidence interval $(P<0.05)$.

\section{RESULTS}

\section{Loss of capsule in cps2J-positive endocarditis isolates}

A total of 288 cps2J-positive isolates were examined for capsular production by the coagglutination test. All 32

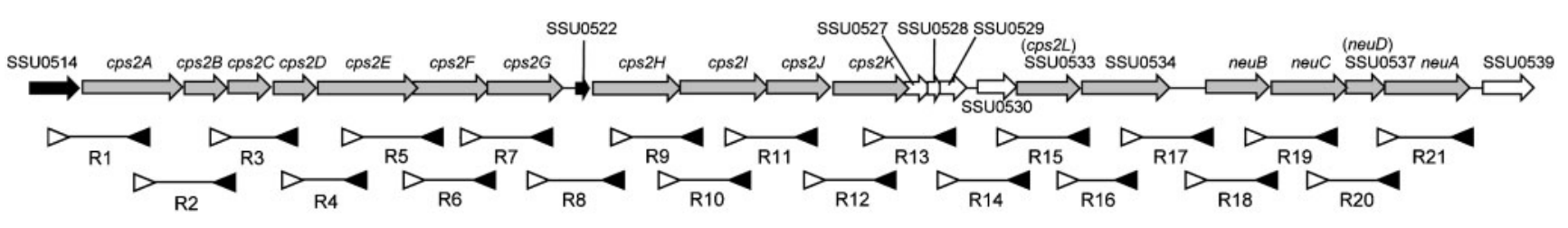

Fig. 1. Genetic organization of the serotype 2 cps locus and positions of primers used for PCR scanning. Grey arrows represent genes putatively involved in capsule synthesis. Black arrows indicate genes encoding hypothetical proteins. White arrows indicate pseudogenes. White and black arrowheads represent forward and reverse primers, respectively. 
isolates from meningitis exhibited agglutination with antiserotype 2 serum, and 4 were also agglutinated with antiserotype 1 serum, indicating that the 28 and 4 isolates were serotypes 2 and $1 / 2$, respectively. In contrast, although $170(66 \%)$ isolates from endocarditis were agglutinated with antiserotype 2 serum, $86(34 \%)$ isolates were not agglutinated with either antiserotype 1 or antiserotype 2 sera, suggesting that these isolates have lost their capsule. To confirm the results, we selected two coagglutinationpositive (P1/7 and NL333) and four coagglutinationnegative (NL146, NL194, NL240 and NL290) isolates and examined them by TEM. As shown in Fig. 2, cells of both coagglutination-positive isolates were surrounded by a thick capsule, whereas no capsular material could be seen in the coagglutination-negative isolates. These results demonstrate that, unlike meningitis isolates, notable numbers of endocarditis isolates lose their ability to produce the capsule.

\section{Structural alterations have occurred in the cps loci of the unencapsulated isolates}

To investigate the genetic backgrounds affecting capsular expression, we randomly selected 43 representative unencapsulated isolates and analysed the genes in their cps loci by PCR scanning. As shown in Supplementary Table S1, available with the online version of this paper, 18 isolates gave no and/or unexpected amplifications with at least one of the primer sets, indicating structural alterations in the cps loci. Nucleotide sequencing of those regions showed that genes in the cps loci of 8 and 10 isolates had deletions and insertions, respectively (Fig. 3). However, for the other 25 isolates, no apparent alteration was found in their cps regions by PCR scanning.

Three isolates (NL157, NL191 and NL280) had a partial deletion in the neuB, cps2E and cps2G genes, respectively, whereas five (NL204, NL217, NL268, NL290 and NL319) had a deletion of multiple genes, as shown in Fig. 3. Among the five isolates above, a large deletion (approx. $17.5 \mathrm{~kb}$ fragment including the neuB-A genes) was found in NL217. In addition, this isolate had a 192 bp deletion in the $c p s 2 F$ gene. In NL204 and NL290, $>3 \mathrm{~kb}$ deletions were found in almost the same region (cps2A-E genes), while the SSU0514-cps2A and $c p s 2 F-G$ regions were lost in NL268 and NL319, respectively.

IS or putative IS elements also disrupted $c p s$ genes (Fig. 3, Supplementary Table S2). The IS elements found in NL85 and NL194 were classified into the ISL3 family, while those of NL155, NL171, NL198, NL201 and NL255 were classified into the IS110 family. Although a putative IS element found in NL184 did not show significant similarity to any other known IS elements, the closest relative was ISTel1 of Thermosynechococcus elongatus BP-1, which belongs to the IS481 family (Supplementary Table S2). However, the sequences inserted in the cps loci of NL176 and NL179 encoded putative reverse transcriptases that constitute group II introns (Michel \& Ferat, 1995).

Notably, most of the deletions and insertions were found in the $c p s 2 A-G$ and neuB-A regions. In particular, $c p s 2 E$ was a hot spot for mutations, as 7 of 10 insertions were found in this gene. On the other hand, the cps2H-SSU0534 region of the isolates analysed in this study was rarely affected.

\section{Unencapsulation increases the ability of S. suis to adhere to porcine and human platelets}

Adherence of bacteria in the bloodstream to platelets on the damaged endocardial surface is thought to be an important mechanism for the initial colonization of cardiac valves (Sullam et al., 1996). To investigate the biological significance of the unencapsulation of $S$. suis in the pathogenesis of infective endocarditis, we compared the ability of encapsulated strain P1/7 and unencapsulated
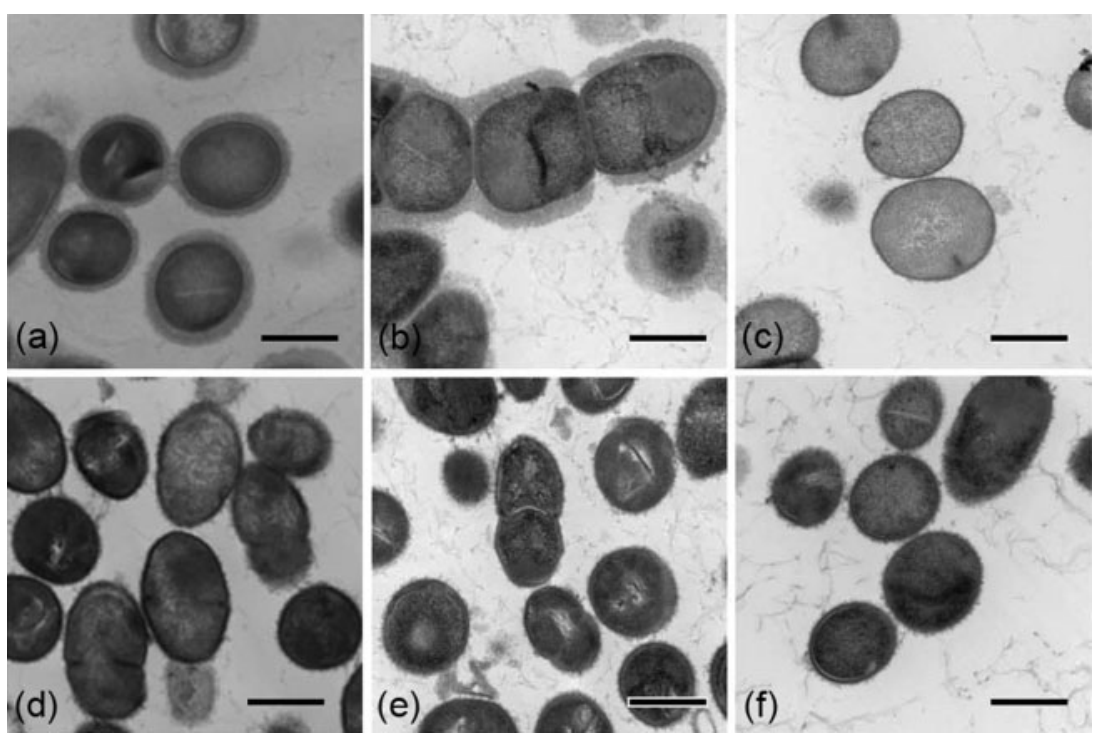

Fig. 2. Transmission electron micrographs of ultrathin sections of $S$. suis strains. Cells of strains P1/7 (a) and NL333 (b) were obviously surrounded by the capsule, while capsular materials were not seen in strains NL146 (c), NL194 (d), NL240 (e) and NL290 (f). Bars, $0.5 \mu \mathrm{m}$. 


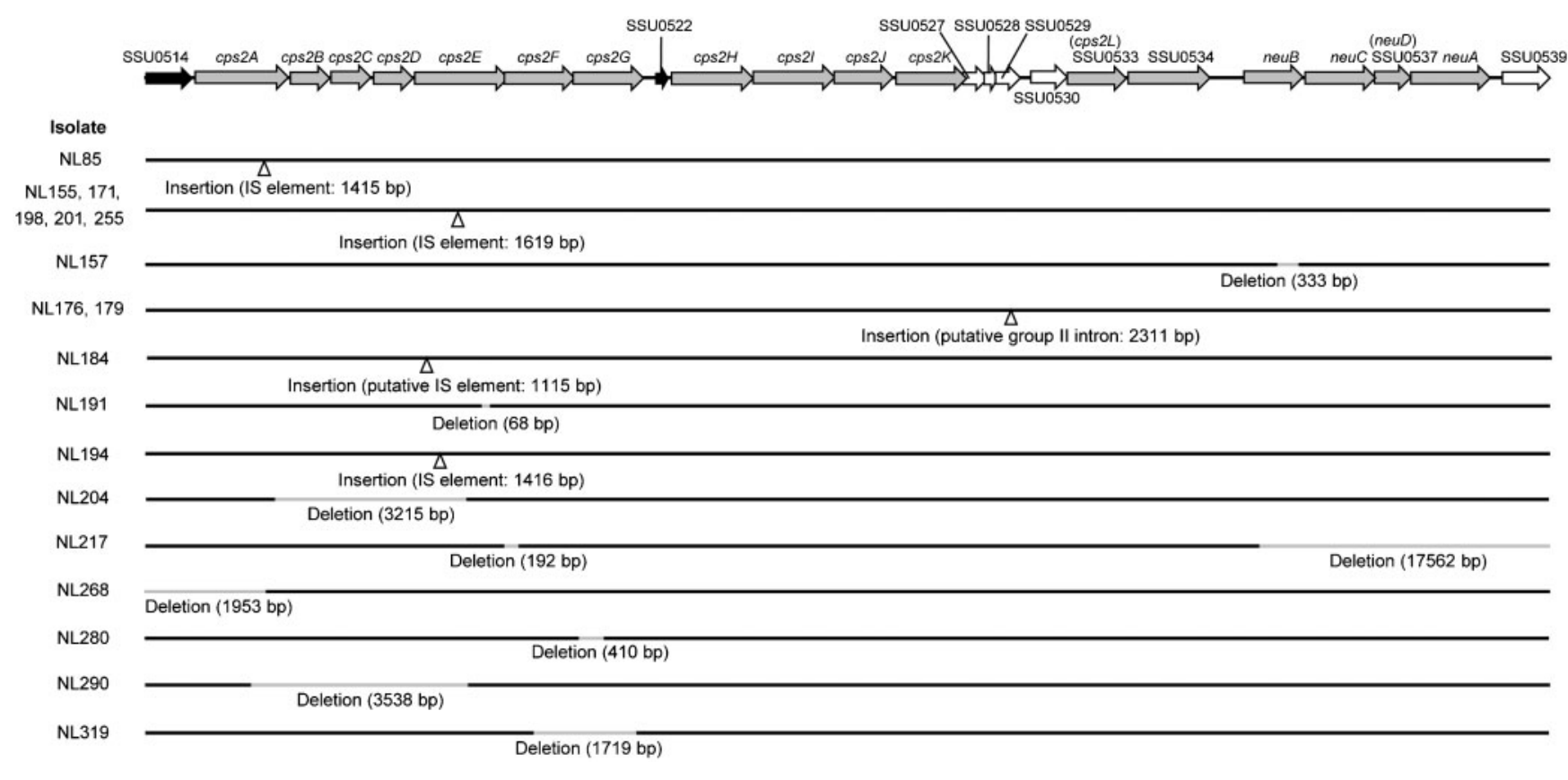

Fig. 3. Positions and types of structural alteration in the cps2 loci of representative unencapsulated endocarditis isolates. Arrowheads indicate positions of insertion. Grey lines indicate deleted regions. Grey arrows represent genes putatively involved in capsule synthesis. Black arrows indicate genes encoding hypothetical proteins. White arrows indicate pseudogenes.

endocarditis isolate NL194 to adhere to porcine platelets. As shown in Fig. 4(a, b), the mean number of attached bacteria per porcine platelet of NL194 $(2.55 \pm 0.3)$ was significantly higher than that of P1/7 $(0.21 \pm 0.07)$ $(P<0.05)$. To evaluate the effect of unencapsulation more precisely, we further analysed the encapsulated serotype 2 strain 89/1591 and its isogenic unencapsulated mutant CPS2B. CPS2B adhered to porcine platelets in greater numbers than 89/1591 (mean no. of attached bacteria per porcine platelet: $2.02 \pm 0.2$ for CPS2B vs $0.44 \pm 0.15$ for $89 /$ 1591, $P<0.05$ ) (Fig. 4a, b). Similar results were obtained when human platelets were used (mean no. of attached bacteria per human platelet: $2.16 \pm 0.22$ for NL194 vs $0.53 \pm 0.38$ for $\mathrm{P} 1 / 7, P<0.05$; and $2.2 \pm 0.37$ for CPS2B vs $0.92 \pm 0.7$ for $89 / 1591, P<0.05$ ) (Fig. $4 \mathrm{c}$ ). These results suggest that unencapsulation facilitates the adherence of $S$. suis to platelets in both swine and humans.

\section{DISCUSSION}

In addition to the molecular basis for serotyping in S. suis (Higgins \& Gottschalk, 2006), the polysaccharide capsule has been shown to be an important virulence factor of this pathogen. Compared with the parent strains, the isogenic unencapsulated mutants of $S$. suis serotype 2 were more susceptible to phagocytosis by both macrophages and neutrophils (Benga et al., 2008; Chabot-Roy et al., 2006; Charland et al., 1998; Smith et al., 1999a). Moreover, capsule loss reduced the virulence of $S$. suis in both mouse and swine models of infection (Charland et al., 1998; Smith et al., 1999a). In contrast, Salasia et al. (1995) and Benga et al. (2004, 2005) reported that isogenic unencapsulated mutants showed increased adherence to various types of cells, including porcine endothelial cells and human epithelial cells, when compared with the parent serotype 2 strains. Esgleas et al. (2005) also demonstrated that an unencapsulated mutant bound to extracellular matrix proteins to a higher degree than its parental encapsulated serotype 2 strain.

In this study, we demonstrated capsule loss in a notable number of $S$. suis isolates from pigs with endocarditis. In agreement with previous studies, the unencapsulated mutants showed a higher degree of adherence to porcine platelets than the encapsulated strains. Bacterial adherence to platelets is considered to be a major virulence determinant in the pathogenesis of infective endocarditis (Sullam et al., 1996). In association with prior injury or disease of the heart valves, the endothelial or exposed connective tissue surface becomes coated with platelets and fibrin. Subsequently, the circulating micro-organisms adhere to and colonize the platelet vegetation, and the colonies are typically encased in more platelets and fibrin, forming the primary infectious lesion or septic vegetation (Herzberg, 1996). Thus, the frequent isolation of unencapsulated isolates from porcine endocarditis might result from the enhanced ability of these isolates to form septic vegetations.

Recently, Tanabe et al. (2010) reported that a capsulardeficient $S$. suis mutant had acquired the capacity to form a thick biofilm, which was not observed in the parent strain. 
(a)

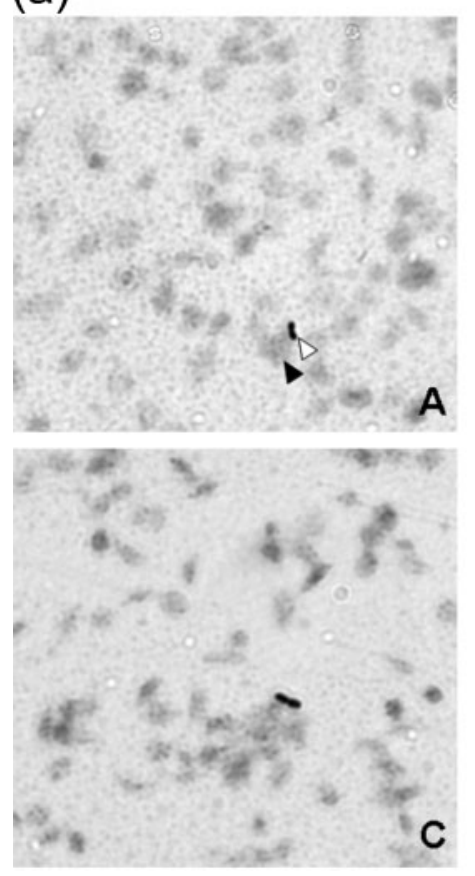

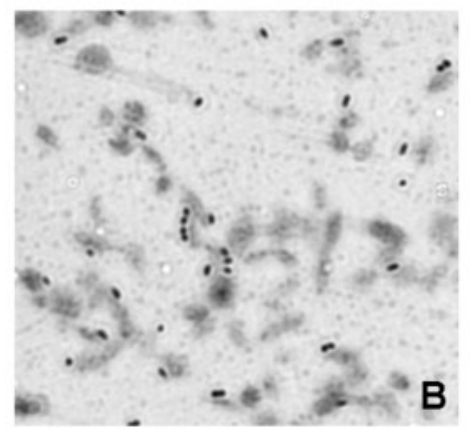

(b)

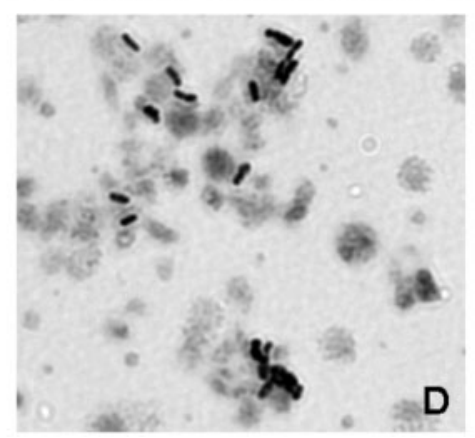

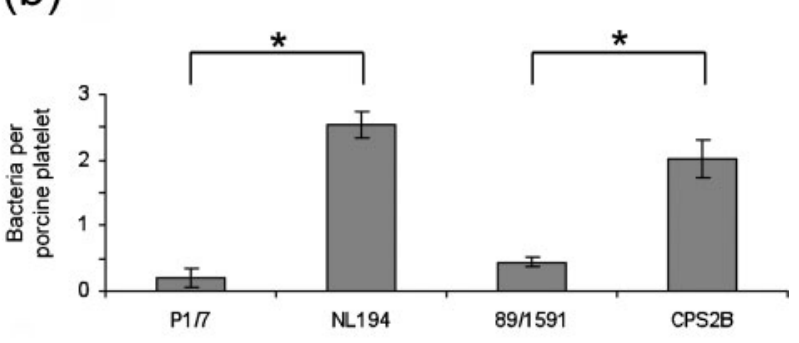

(c)

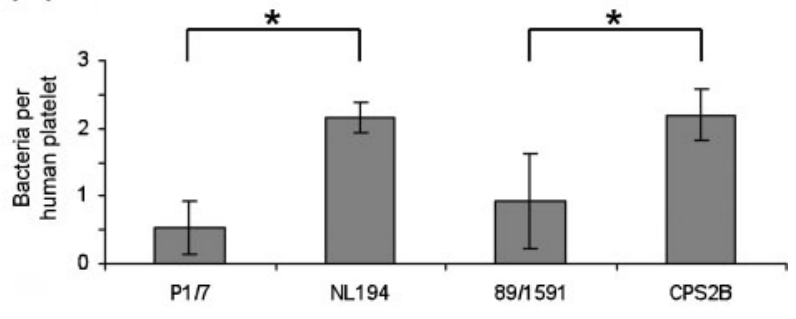

Fig. 4. Bacterial adherence to porcine and human platelets. (a) Adherence of encapsulated and unencapsulated S. suis strains/ isolate to porcine platelets. Bacterial cells and platelets were stained with $5 \%$ Giemsa solution and observed by light microscopy: A, encapsulated serotype 2 strain P1/7; B, unencapsulated endocarditis isolate NL194; C, encapsulated serotype 2 strain 89/1591; D, isogenic unencapsulated mutant of strain 89/1591 (CPS2B). Black and white arrowheads indicate platelets and bacterial cells, respectively. Images taken at $\times 1000$ magnification. (b, c) Ability of $S$. suis strains and isolate NL194 to adhere to porcine (b) and human (c) platelets. Asterisks indicate significant differences $(P<0.05)$.

Although the exact role of biofilm formation in S. suis infections is unclear, such a property may allow the bacteria to become persistent colonizers and to resist clearance by the host immune system. The biofilm-positive phenotype of unencapsulated mutants therefore might give them further advantage in forming cardiac vegetations in swine.

In this study, increased adherence of the unencapsulated isolates to human platelets was also observed. Although human cases of endocarditis caused by unencapsulated S. suis have not yet been reported, an unencapsulated isolate from the blood of an endocarditis patient has been described for group B Streptococcus (GBS) (Sellin et al., 1992). Therefore, our results may suggest the potential of unencapsulated $S$. suis to cause infective endocarditis in humans.

Because encapsulated isolates were retrieved from $66 \%$ of the porcine endocarditis cases analysed in this study, even in cases from which unencapsulated S. suis were isolated, it is unknown whether all bacterial cells present in the vegetations were unencapsulated or whether only a subpopulation of infected bacteria had lost the capsule and the unencapsulated cells established a footing as the first colonizers for further colonization of encapsulated cells. It is also unclear whether unencapsulation occurred after invasion of the bloodstream by bacteria or whether unencapsulated strains can be transmitted among different pigs and farms. Interestingly, we found five isolates (NL155, NL171, NL198, NL201 and NL255) with almost the same IS elements $(99.8-100 \%$ identical at the nucleotide level) at the same position of cps2E (Fig. 3, Supplementary Table S2). Among them, NL155, NL171, NL198 and NL201 were isolated from different pigs in the same prefecture, while NL255 was isolated from the adjacent area. This strongly suggests the transmission of unencapsulated S. suis strains among pigs and farms, although we cannot rule out the possibility that the insertion position was the preferential site for the IS elements and insertion events occurred independently in each isolate. It is of note that most of the deletions and insertions found in the unencapsulated isolates occurred in the cps $2 A-G$ region. Among the genes in this region, $c p s 2 E$ was the most frequently altered. This may support the notion that $\operatorname{cps} 2 E$ and its flanking regions are hot spots for structural alteration.

No apparent deletion or insertion was detected by PCR scanning in the 25 unencapsulated isolates. During preparation of this paper, Willenborg et al. (2011) reported that catabolite control protein A (CcpA) of S. suis is necessary for capsular expression and that deletion of $\operatorname{ccp} A$ resulted in significant reduction of capsule thickness. However, the deduced amino acid sequences of $c c p A$ of 
the 25 unencapsulated isolates were $99.4-100 \%$ identical to that of strain P1/7 and $99.7-100 \%$ identical to that of strain 89/1591 (our unpublished observations), suggesting that $\operatorname{ccp} A$ of the unencapsulated isolates was intact. Therefore, their cps loci may be affected by point mutations that could not be detected by PCR scanning. Alternatively, it is also conceivable that their capsular expression was negatively regulated by unknown mechanisms. In fact, phase variation of capsular expression has previously been reported in a GBS isolate. In this case, although the original strain isolated from the blood of a patient with endocarditis was unencapsulated, encapsulated variants could be recovered after Percoll gradient centrifugation (Sellin et al., 1992). Similar phase variations have been found in other Gram-positive and -negative bacteria (van der Woude \& Bäumler, 2004). Although such variations have not been reported in S. suis, capsular expression might be reversible in some unencapsulated S. suis isolates. Moreover, even in unencapsulated isolates with mutations affecting capsular expression, capsule production could be restored by acquiring functional genes via horizontal gene transfer, additional point mutations or the excision of inserted sequences. Further studies to investigate the above possibilities will provide additional insights into the role of the capsule in the pathogenesis of S. suis infection.

It is noteworthy that the cps loci of NL176 and NL179 were similarly disrupted by the insertion of a putative group II intron within SSU0529. Because SSU0529 is considered to be a pseudogene, it cannot be assumed that the disruption of SSU0529 itself caused the loss of capsular production in these isolates. Thus, in these two isolates, additional point mutations and/or negative regulation of capsular expression might occur in addition to the insertion events. Alternatively, the expression of downstream genes might be influenced by a polar effect caused by insertion of the group II intron.

In conclusion, we have demonstrated that approximately one-third of the endocarditis isolates from swine that we examined had lost the ability to produce capsules and that unencapsulation enhanced bacterial adherence to porcine and human platelets. Although the capsule is thought to be an important virulence factor for $S$. suis, our results suggest that loss of capsule production may be beneficial for S. suis in causing infective endocarditis.

\section{ACKNOWLEDGEMENTS}

Part of this study was supported by the Japan Society for the Promotion of Science with a Grant-in-Aid for Scientific Research (C) (23580420). We thank Masahiro Kusumoto for helpful suggestions on the sequence analysis. We also thank the Japan Red Cross Society for kindly supplying human platelets.

\section{REFERENCES}

Benga, L., Goethe, R., Rohde, M. \& Valentin-Weigand, P. (2004). Non-encapsulated strains reveal novel insights in invasion and survival of Streptococcus suis in epithelial cells. Cell Microbiol 6, 867-881.

Benga, L., Friedl, P. \& Valentin-Weigand, P. (2005). Adherence of Streptococcus suis to porcine endothelial cells. J Vet Med B Infect Dis Vet Public Health 52, 392-395.

Benga, L., Fulde, M., Neis, C., Goethe, R. \& Valentin-Weigand, P. (2008). Polysaccharide capsule and suilysin contribute to extracellular survival of Streptococcus suis co-cultivated with primary porcine phagocytes. Vet Microbiol 132, 211-219.

Chabot-Roy, G., Willson, P., Segura, M., Lacouture, S. \& Gottschalk, M. (2006). Phagocytosis and killing of Streptococcus suis by porcine neutrophils. Microb Pathog 41, 21-32.

Charland, N., Harel, J., Kobisch, M., Lacasse, S. \& Gottschalk, M. (1998). Streptococcus suis serotype 2 mutants deficient in capsular expression. Microbiology 144, 325-332.

Esgleas, M., Lacouture, S. \& Gottschalk, M. (2005). Streptococcus suis serotype 2 binding to extracellular matrix proteins. FEMS Microbiol Lett 244, 33-40.

Gottschalk, M., Higgins, R., Jacques, M., Mittal, K. R. \& Henrichsen, J. (1989). Description of 14 new capsular types of Streptococcus suis. J Clin Microbiol 27, 2633-2636.

Gottschalk, M., Higgins, R. \& Jacques, M. (1993). Production of capsular material by Streptococcus suis serotype 2 under different growth conditions. Can J Vet Res 57, 49-52.

Gottschalk, M., Segura, M. \& Xu, J. (2007). Streptococcus suis infections in humans: the Chinese experience and the situation in North America. Anim Health Res Rev 8, 29-45.

Han, D. U., Choi, C., Ham, H. J., Jung, J. H., Cho, W. S., Kim, J., Higgins, R. \& Chae, C. (2001). Prevalence, capsular type and antimicrobial susceptibility of Streptococcus suis isolated from slaughter pigs in Korea. Can J Vet Res 65, 151-155.

Herzberg, M. C. (1996). Platelet-streptococcal interactions in endocarditis. Crit Rev Oral Biol Med 7, 222-236.

Higgins, R. \& Gottschalk, M. (1990). An update on Streptococcus suis identification. J Vet Diagn Invest 2, 249-252.

Higgins, R. \& Gottschalk, M. (2006). Streptococcal diseases. In Diseases of Swine, pp. 769-783. Edited by B. E. Straw, J. J. Zimmerman, S. D’Allaires \& D. J. Taylor. Ames, Iowa: Blackwell Publishing.

Holden, M. T., Hauser, H., Sanders, M., Ngo, T. H., Cherevach, I., Cronin, A., Goodhead, I., Mungall, K., Quail, M. A. \& other authors (2009). Rapid evolution of virulence and drug resistance in the emerging zoonotic pathogen Streptococcus suis. PLoS ONE 4, e6072.

Hoshinoo, K., Sasaki, K., Tanaka, A., Corbeil, L. B. \& Tagawa, Y. (2009). Virulence attributes of Histophilus somni with a deletion mutation in the ibpA gene. Microb Pathog 46, 273-282.

Jacques, M., Gottschalk, M., Foiry, B. \& Higgins, R. (1990). Ultrastructural study of surface components of Streptococcus suis. J Bacteriol 172, 2833-2838.

Kataoka, Y., Sugimoto, C., Nakazawa, M., Morozumi, T. \& Kashiwazaki, M. (1993). The epidemiological studies of Streptococcus suis infections in Japan from 1987 to 1991. J Vet Med Sci 55, 623-626.

Katsumi, M., Kataoka, Y., Takahashi, T., Kikuchi, N. \& Hiramune, T. (1997). Bacterial isolation from slaughtered pigs associated with endocarditis, especially the isolation of Streptococcus suis. J Vet Med Sci 59, 75-78.

Mackie, E. B., Brown, K. N., Lam, J. \& Costerton, J. W. (1979). Morphological stabilization of capsules of group B streptococci, types Ia, Ib, II, and III, with specific antibody. J Bacteriol 138, 609-617.

Michel, F. \& Ferat, J. L. (1995). Structure and activities of group II introns. Annu Rev Biochem 64, 435-461. 
Mogollon, J. D., Pijoan, C., Murtaugh, M. P., Kaplan, E. L., Collins, J. E. \& Cleary, P. P. (1990). Characterization of prototype and clinically defined strains of Streptococcus suis by genomic fingerprinting. J Clin Microbiol 28, 2462-2466.

Ochman, H., Gerber, A. S. \& Hartl, D. L. (1988). Genetic applications of an inverse polymerase chain reaction. Genetics 120, 621-623.

Okura, M., Osaki, M., Fittipaldi, N., Gottschalk, M., Sekizaki, T. \& Takamatsu, D. (2011). The minor pilin subunit Sgp2 is necessary for assembly of the pilus encoded by the srt $G$ cluster of Streptococcus suis. J Bacteriol 193, 822-831.

Okwumabua, O., O'Connor, M. \& Shull, E. (2003). A polymerase chain reaction (PCR) assay specific for Streptococcus suis based on the gene encoding the glutamate dehydrogenase. FEMS Microbiol Lett 218, 79-84.

Salasia, S. I., Lämmler, C. \& Herrmann, G. (1995). Properties of a Streptococcus suis isolate of serotype 2 and two capsular mutants. Vet Microbiol 45, 151-156.

Sekizaki, T., Otani, Y., Osaki, M., Takamatsu, D. \& Shimoji, Y. (2001). Evidence for horizontal transfer of SsuDAT1I restriction-modification genes to the Streptococcus suis genome. J Bacteriol 183, 500-511.

Sellin, M., Linderholm, M., Norgren, M. \& Håkansson, S. (1992). Endocarditis caused by a group B Streptococcus strain, type III, in a nonencapsulated phase. J Clin Microbiol 30, 2471-2473.

Silva, L. M., Baums, C. G., Rehm, T., Wisselink, H. J., Goethe, R. \& Valentin-Weigand, P. (2006). Virulence-associated gene profiling of Streptococcus suis isolates by PCR. Vet Microbiol 115, 117-127.

Slater, J. D., Allen, A. G., May, J. P., Bolitho, S., Lindsay, H. \& Maskell, D. J. (2003). Mutagenesis of Streptococcus equi and Streptococcus suis by transposon Tn917. Vet Microbiol 93, 197-206.

Smith, H. E., Damman, M., van der Velde, J., Wagenaar, F., Wisselink, H. J., Stockhofe-Zurwieden, N. \& Smits, M. A. (1999a). Identification and characterization of the cps locus of Streptococcus suis serotype 2: the capsule protects against phagocytosis and is an important virulence factor. Infect Immun 67, 1750-1756.

Smith, H. E., van Bruijnsvoort, L., Buijs, H., Wisselink, H. J. \& Smits, M. A. (1999b). Rapid PCR test for Streptococcus suis serotype 7. FEMS Microbiol Lett 178, 265-270.

Smith, H. E., Veenbergen, V., van der Velde, J., Damman, M., Wisselink, H. J. \& Smits, M. A. (1999c). The cps genes of Streptococcus suis serotypes 1, 2, and 9: development of rapid serotype-specific PCR assays. J Clin Microbiol 37, 3146-3152.

Smith, H. E., de Vries, R., van't Slot, R. \& Smits, M. A. (2000). The cps locus of Streptococcus suis serotype 2: genetic determinant for the synthesis of sialic acid. Microb Pathog 29, 127-134.

Sullam, P. M., Bayer, A. S., Foss, W. M. \& Cheung, A. L. (1996). Diminished platelet binding in vitro by Staphylococcus aureus is associated with reduced virulence in a rabbit model of infective endocarditis. Infect Immun 64, 4915-4921.

Tanabe, S., Bonifait, L., Fittipaldi, N., Grignon, L., Gottschalk, M. \& Grenier, D. (2010). Pleiotropic effects of polysaccharide capsule loss on selected biological properties of Streptococcus suis. Can J Vet Res 74, 65-70.

van der Woude, M. W. \& Bäumler, A. J. (2004). Phase and antigenic variation in bacteria. Clin Microbiol Rev 17, 581-611.

Wertheim, H. F., Nghia, H. D., Taylor, W. \& Schultsz, C. (2009). Streptococcus suis: an emerging human pathogen. Clin Infect Dis $\mathbf{4 8}$, 617-625.

Willenborg, J., Fulde, M., de Greeff, A., Rohde, M., Smith, H. E., Valentin-Weigand, P. \& Goethe, R. (2011). Role of glucose and CcpA in capsule expression and virulence of Streptococcus suis. Microbiology 157, 1823-1833. 